\title{
One-way waveguide and strong photon-photon interaction in nanophotonic structures
}

\author{
Shanhui Fan, Jung-Tsung Shen, Zongfu Yu, Georgios Veronis, and Zheng Wang \\ Ginzton Laboratory, Stanford University, Stanford, CA 94305 USA
}

\begin{abstract}
New opportunities of manipulating light propagation as well as controlling light-matter interaction arise with the use of nanophotonic structures. Here we discuss two recent examples of such opportunities: a one-way electromagnetic waveguide that fundamentally suppresses the effects of disorder-induced backscattering, as well as the possibility of creating strong photon-photon interactions at a few photon level with the use of photonic crystals.
\end{abstract}

\section{INTRODUCTION}

We consider some of the new optical effects that arise in nanophotonic structures. In particular, we show that by embedding a two-level atom into a photonic crystal waveguide, one can create strong photon-photon interaction and correlations at few photon-level [1][2]. Such a capability is potentially important for quantum information processing. We also show that a one-way waveguide can form at the interface between a dielectric photonic crystal, and a plasmonic freeelectron metal subject to a static DC magnetic field [3]. In such a waveguide, disorder-induced backscattering is completely suppressed. Such a one-way waveguide represents a new regime of photon transport in nanostructures, and can have important implications for on-chip integrated photonic circuits.

\section{STRONGLY-CORRELATED TWO-PHOTON TRANSPORT IN PHOTONIC CRYSTAL WAVEGUIDES}

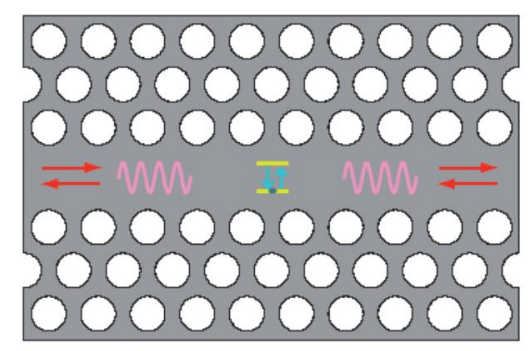

Fig. 1. A two-level system coupled to a photonic crystal waveguide in which the photons, shown as wiggly waves, propagate in each direction.
Creating a strong photon-photon interaction at the fewphoton level is of great interest for quantum information sciences. In atomic gases, such an interaction can be accomplished either with systems exhibiting electromagnetically induced transparency (EIT) $[3,4]$, or by reaching the strong-coupling regime of a two-level atom in a high-Q cavity [5]. However, in an on-chip, solid-state environment, which is crucial for practical applications, there have been significant challenges in implementing these concepts. For example, it is difficult to create the long-lifetime dark state, which is required for EIT effects, in most practical solid-state environments [6]. While the strongcoupling regime has been reached by placing a quantum dot in a high-Q photonic crystal microcavity $[7,8]$, doing so requires very accurate tuning of both the electronic and optical resonances to ensure simultaneous spectral and spatial overlaps [9].

Here we propose and analyze in detail an alternative scheme to create strong photon-photon interaction [1]. Our approach exploits a unique one-dimensional feature for photon states in many nano-photonic structures. In a photonic crystal with a complete photonic band gap, for example, a line-defect waveguide forms a true onedimensional continuum for photons, since there are no other states within the gap. Here we show that by coupling a two-level system to such a continuum, strong photon-photon interactions can be created (Fig. 1). In this system, the strong interaction arises from the fact that, in a one-dimensional system, the re-emitted and scattered waves from the atom inevitably interfere with the incident waves [2]. Moreover, since the atom, intuitively speaking, can at most absorb only one photon at a time, the transport properties of multiphotons are strongly correlated.

Compared with previous solid-state approaches, our scheme does not require the presence of a long-lifetime dark state. Neither does this scheme necessitate detailed spectral tuning or spatial control of the two-level system, since it operates in the weak-coupling regime, and thus the one-dimensional continuum can be broadband. Moreover, the Hamiltonian of the system actually describes an exact photonic analogue of the Kondo effect, which is important for processing electronic quantum bits [10]. Our approach may 
therefore open a new avenue toward practical photonbased quantum information processing on-chip.

\section{ONE-WAY ELECTROMAGNETIC WAVEGUIDE}

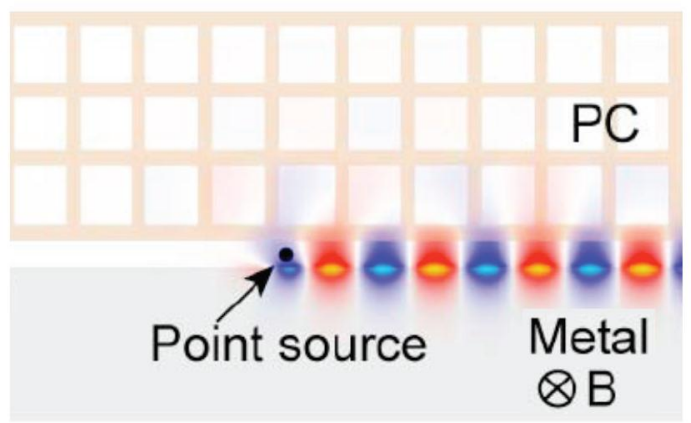

Fig. 2. One-way waveguide formed at the interface between a dielectric photonic crystal and a free-electron plasmonic subject to a static out-of-plane magnetic field. In such a waveguide, a point source radiates only to one direction but not the other.

Understanding and controlling the effects of disorders on wave propagation are both of fundamental interest, and are becoming increasingly important in practical nano-device applications. In general, the effects of disorders are drastically influenced by time-reversal symmetry properties of a system. In reciprocal nanophotonic systems, which include most photonic crystals and plasmonic waveguides, at any given frequency for each forward propagating mode, there is a corresponding backward propagating mode with identical mode shapes. In the presence of disorders, these two modes, having maximal modal overlap, always scatter into each other, resulting in backreflection. Such back-reflection can be particularly worrisome for slow light systems, which are of current interest for optical signal processing applications. As the group velocity $\mathrm{v}_{\mathrm{g}}$ is reduced, the back reflection increases as $1 / \mathrm{v}_{\mathrm{g}}{ }^{2}$, and in slow light systems may dominate over all other loss mechanisms $[12,13]$.

In systems with broken time-reversal symmetry, the effect of disorders can be suppressed with the use of a one-way waveguide [14]. Such a waveguide supports a single forward propagating mode in a given frequency range, while having neither radiation nor backward propagation modes in the same range. For electronic waves, an example of a one-way waveguide is the edge states for a two-dimensional electron gas in the quantum hall regime [15]. Here we introduce a mechanism for creating a one-way electromagnetic waveguide [3]. The waveguide is formed at the interface between a dielectric photonic crystal that is assumed to be reciprocal, and a free-electron plasmonic metal under a static magnetic field along the direction perpendicular to the plane of propagation. (Fig. 2) We show that such a waveguide supports a one-way waveguide mode, provided that the surface plasmon frequency of the metal surface lies within the band gap of the photonic crystal. In such a system, the presence of the band gap is intrinsic to the photonic crystal and the metal itself, independent of time-reversal symmetry breaking. Hence the size of band gap can be made very large and can survive significant disorders [16]. As a proof of concept, we present direct numerical evidence demonstrating suppression of disorder effects. We also show that the nature of waveguide-cavity interaction, which is at the heart of many integrated devices, can be fundamentally altered. Such a waveguide therefore represents a complete new regime of light transport with important implications for on-chip integrated photonic circuits

\section{REFERENCES}

1. J. T. Shen and S. Fan, Phys. Rev. Lett. 98, 153003 (2007).

2. J. T. Shen and S. Fan, Opt. Lett. 30, 2001 (2005).

3. Z. Yu et al (submitted).

4. S. E. Harris et al., Phys. Rev. Lett. 81, 3611 (1998).

5. A. Imamoglu et al., Phys. Rev. Lett. 79, 1467 (1997).

6. K. M. Birnbaum et al., Nature 436, 87 (2005).

7. A.V. Turukhin et al., Phys. Rev. Lett. 88,023602 (2001).

8. J. P. Reithmaier et al., Nature 432, 197 (2004).

9. T. Yoshie et al., Nature 432, 200 (2004).

10. A. Badolato et al., Science 308, 1158 (2005).

11. D. M. Zumbuhl et al., Phys. Rev. Lett. 93, 256801 (2004).

12. S. Hughes et al, Phys. Rev. Lett. 94, 033903 (2005).

13. S. G. Johnson et al, Appl. Phys. B 81, 283 (2005).

14. F. D. M. Haldane and S. Raghu, condmat/0503588.

15. S. Datta, Electronic Transport in Mesoscopic Systems (Cambridge University Press, Cambridge, UK, 1995).

16. S. Fan et al, J. Appl. Phys. 78, 1415 (1995). 